\section{Ranibizumab for the treatment of choroidal neovascularisation secondary to pathological myopia: interim analysis of the REPAIR study}

This article has been corrected since Advance Online Publication and a corrigendum is also printed in this issue.

\begin{abstract}
Aims To evaluate the efficacy and safety of intravitreal ranibizumab in patients with choroidal neovascularisation secondary to pathological myopia (myopic CNV). Data are from a pre-planned, 6-month interim analysis. Methods Phase II, open-label, single arm, multicentre, 12-month study, recruiting patients (aged $\geq 18$ years) with active primary or recurrent subfoveal or juxtafoveal myopic $\mathrm{CNV}$, with a best-corrected visual acuity (BCVA) score of 24-78 Early Treatment Diabetic Retinopathy Study (ETDRS) letters in the study eye and a diagnosis of high myopia of at least -6 dioptres.

Patients received $0.5 \mathrm{mg}$ ranibizumab administered intravitreally to the study eye, followed by monthly injections given as needed (based on a predefined algorithm) for up to $\mathbf{1 1}$ months.

Results At 6 months, mean BCVA improved from baseline by $\mathbf{1 2 . 2}$ letters, as did central macular thickness (in this interim analysis defined as a measure of either central subfield macular thickness or centre point macular thickness) from baseline by $108 \mu \mathrm{m}$ in the 48 study eyes of 48 patients. Fewer patients had centre-involving intraretinal

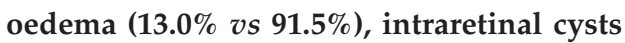

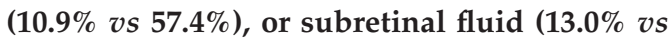
$66.0 \%$ ) at 6 months than at baseline. Patients received a mean of 1.9 retreatments, were
\end{abstract}

A Tufail ${ }^{1}$, PJ Patel ${ }^{1}$, S Sivaprasad ${ }^{2}$, W Amoaku ${ }^{3}$, AC Browning ${ }^{4}$, M Cole ${ }^{5}, \mathrm{R}$ Gale ${ }^{6}$, S George ${ }^{7}$, AJ Lotery ${ }^{8}, \mathrm{M} \mathrm{Majid}^{9}, \mathrm{M} \mathrm{McKibbin}^{10}$, G Menon ${ }^{11}$, Y Yang ${ }^{12}, C_{\text {Andrews }}^{13}, C$ Brittain $^{13}$ and A Osborne ${ }^{13}$

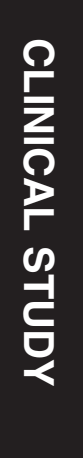

\author{
${ }^{1}$ Moorfields Eye Hospital, \\ London, UK \\ ${ }^{2}$ Kings College Hospital, \\ London, UK \\ ${ }^{3}$ Queens Medical Centre, \\ Nottingham, UK \\ ${ }^{4}$ Royal Victoria Infirmary, \\ Newcastle, UK \\ ${ }^{5}$ Torbay Hospital, Torquay, UK \\ ${ }^{6}$ York District Hospital, York, UK \\ ${ }^{7}$ Western Eye Hospital, London, \\ UK \\ ${ }^{8} \mathrm{Clinical}$ Neurosciences and \\ Experimental Sciences, Faculty \\ of Medicine Division, University \\ of Southampton, Southampton, \\ UK \\ ${ }^{9}$ Bristol Eye Hospital, Bristol, UK \\ ${ }^{10}$ St James's University Hospital, \\ Leeds, UK \\ ${ }^{11}$ Frimley Park Hospital, Frimley, \\ UK \\ ${ }^{12}$ Wolverhampton Eye Infirmary، \\ Wolverhampton, UK \\ ${ }^{13}$ Novartis Pharmaceuticals Ltd., \\ Surrey, UK
}

satisfied with ranibizumab treatment, and well being was maintained. No new safety signals were identified.

Conclusions Results from the planned interim analysis support the role of ranibizumab in the treatment of myopic $\mathrm{CNV}$, with excellent efficacy achieved with a low number of injections and few serious adverse events. Eye (2013) 27, 709-715; doi:10.1038/eye.2013.8; published online 1 March 2013

Keywords: myopia; degenerative; choroidal neovascularisation; clinical trial; ranibizumab

\section{Introduction}

Pathological myopia, which affects $\sim 2-4 \%$ of Caucasians and $9-21 \%$ of Asian populations, is a major cause of blindness in the developed countries. ${ }^{1-3}$ The irreversible central vision loss that can occur in patients with pathological myopia most commonly results from the complication of choroidal neovascularisation (CNV). CNV is detectable in $4-11 \%$ of affected eyes ${ }^{4}$ with pathological myopia and occurs predominantly in those younger than 50 years of age, with almost $90 \%$ of eyes developing severe visual loss $(\leq 20 / 200)$ after 5-10 years. ${ }^{5,6}$ Furthermore, in patients with established myopic $\mathrm{CNV}$ in one eye, about a third will develop CNV in
Correspondence:

A Tufail, Moorfields Eye

Hospital, 162 City Road

London EC1V 2PD, UK

E-mail: Adnan.Tufail@

moorfields.nhs.uk

Previous presentation: 1. American Academy of Ophthalmology Annual Meeting 2011.

2. The Association for Research in Vision and Ophthalmology

Annual Meeting 2012.

3. The Royal College of Ophthalmology Annual Meeting 2012

Received: 28 June 2012 Accepted in revised form: 26 November 2012

Published online: 1 March 2013 
the fellow eye within 8 years, ${ }^{7}$ which would lead to significant morbidity of the individual's binocular visual function in their productive years.

Thermal laser for myopic CNV is of limited value, as treatment of subfoveal lesions induces severe immediate visual loss, whereas treatment of juxta- and extrafoveal lesions is complicated by a high recurrence rate and longterm expansion of the laser scar. ${ }^{3,8}$ The long-term results for verteporfin-PDT (vPDT) have also been disappointing. ${ }^{5}$ Data from the large $(n=120)$, multinational, randomized, VIP study showed a significant benefit for vPDT over sham therapy for the primary efficacy end point (avoidance of a loss of eight letters or more from baseline) after 1 year in patients with myopic CNV (72\% vs $44 \%$; $P<0.01) .{ }^{9}$

However, the significance of this benefit was lost at 2 years, $64 \%$ vs $49 \% P=0.11$, although the secondary efficacy end points continued to favour vPDT over sham. ${ }^{10}$

Extrapolating from studies that show superiority of an antivascular endothelial growth factor (anti-VEGF) therapy to vPDT for neovascular age-related macular degeneration (AMD, (ANCHOR, $\left.{ }^{11} \mathrm{ABC}^{12}\right)$ ), clinicians have started using anti-VEGF off-label for the treatment of myopic CNV. Recent findings from multiple small case series have shown promise for anti-VEGF agents in myopic CNV, although ideally randomized, controlled trials are needed to confirm results. ${ }^{13}$ The one large prospective series published supports the benefit of antiVEGF therapy; ${ }^{14}$ it is of a $3+$ prn design where patients could have received pegaptanib and vPDT previously. Despite these published case series, availability of antiVEGF for myopic CNV is limited in the United Kingdom and further evidence to support its use is required. In the United Kingdom, retina specialists felt that a randomized, controlled trial would be difficult to recruit, as some patients would have to be randomized to vPDT, which is perceived by patients and clinicians to be inferior. REPAIR is the largest prospective study so far of ranibizumab in myopic CNV. It was designed to evaluate the efficacy and safety of intravitreal ranibizumab $(0.5 \mathrm{mg})$ in patients with myopic CNV using an individualized (pro re nata (PRN)) dosing schedule as needed. This manuscript reports an interim analysis of the REPAIR study, which was pre-planned for when $75 \%$ of patients had completed 6 months of the study (eight visits). Previous case series evaluating the role of anti-VEGF in myopic CNV have suggested a more self-limiting disease course, and therefore these interim data are of clinical use to the community.

\section{Materials and methods}

\section{Patients and design}

This is a phase II, open-label, single arm, multicentre, 12-month study conducted in 12 centres in the United
Kingdom. The study was conducted in accordance with the Declaration of Helsinki and guidelines for Good Clinical Practice, and ethics approval was obtained from the relevant ethics committees. All patients provided written informed consent prior to enrolment. We certify that all applicable institutional and governmental regulations concerning the use of human volunteers were followed during this research.

The study is registered at ClinicalTrials.gov, identifier: NCT01037348.

Patients aged $\geq 18$ years with active primary or recurrent subfoveal or juxtafoveal myopic CNV were included in the study. Eligible patients were also required to have a best-corrected visual acuity (BCVA) score of 24-78 Early Treatment Diabetic Retinopathy Study (ETDRS) letters in the study eye. Myopia was defined in the study entry criteria as a spherical equivalent of 6 dioptres of myopia (preoperatively if the eye had previously undergone cataract or refractive surgery).

Patients were excluded from participation if they had: a history of any surgical intervention in the study eye within 2 months of the screening visit, current or previous macular laser photocoagulation, treatment with intravitreal steroids, vPDT or anti-VEGF agents in the study eye, or prior treatment in the study eye with external-beam radiation therapy, vitrectomy, or transpupillary thermotherapy. Those with current use or likely need for systemic medications known to be toxic to the lens, retina or optic nerve, concurrent use of systemic anti-VEGF therapy or previous treatment with intravenously administered bevacizumab, concurrent use of chronic non-steroidal anti-inflammatory drugs (NSAIDs) for more than 7 consecutive days, or systemic or topical ocular corticosteroids for $\geq 3$ consecutive days within 6 months prior to baseline were also excluded. Patients who presented with $\mathrm{CNV}$ from causes other than pathological myopia and those with ocular disorders in the study eye that may confound interpretation of study results were not eligible for participation.

Eligible patients received one initial injection of $0.5 \mathrm{mg}$ ranibizumab in $0.05 \mathrm{ml}$ solution administered intravitreally to the study eye by a qualified ophthalmologist experienced in the procedure. The dose was chosen in accordance with the product labelling for treatment of $\mathrm{AMD}^{15}$ and the dose level used in several recent case series in myopic CNV. ${ }^{16-19}$ The initial dose was followed by repeated injections, administered as needed (PRN) following monthly monitoring with Spectral Domain Optical Coherence Tomography (SD OCT), but no more frequently than every 28 days, for upto a further 11 months. The algorithm used to determine the need for retreatment is presented in Figure 1. There was no control treatment in this study. 


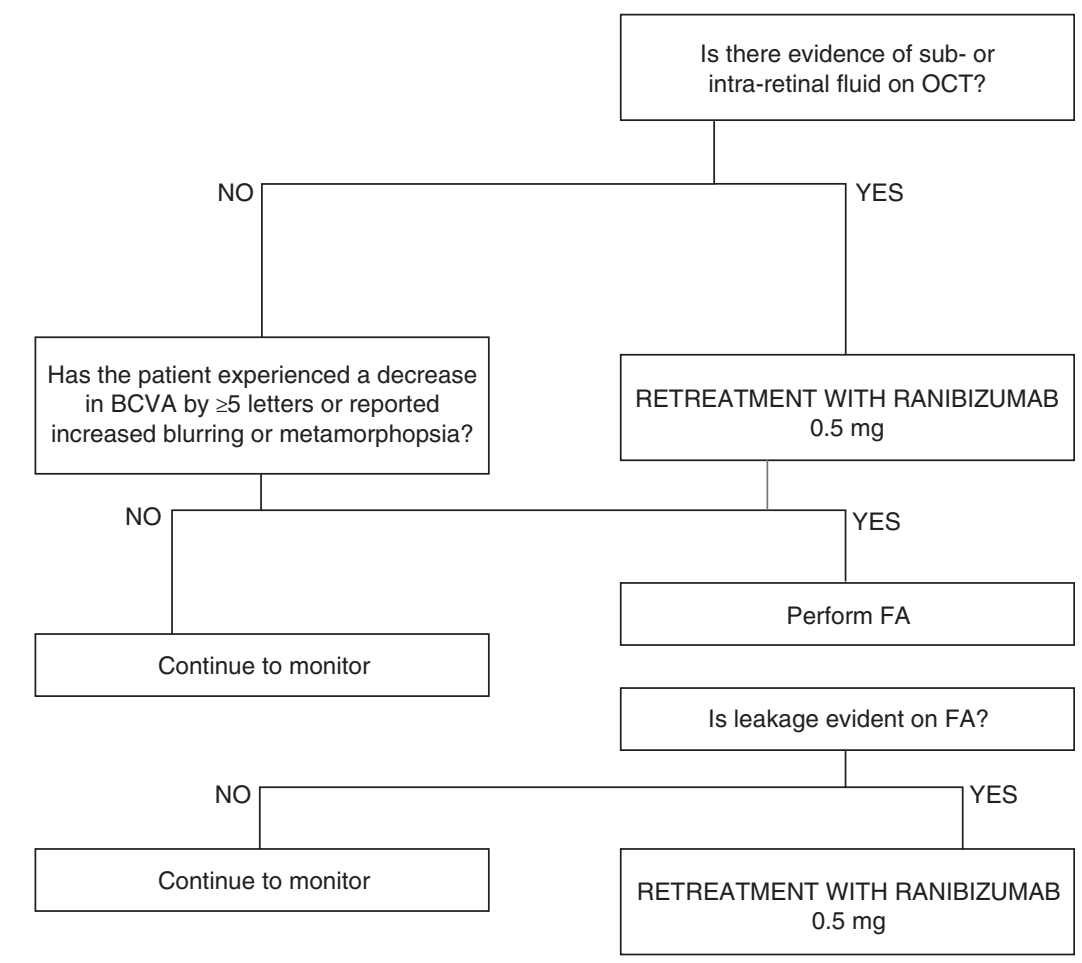

Figure 1 REPAIR retreatment algorithm.

\section{Assessments}

Visits to assess efficacy and safety were scheduled at 1-month intervals during the treatment period.

The primary objective of this study was the evaluation of mean change in BCVA (initial testing distance of $4 \mathrm{~m}$ ) from baseline to month 12 . The key secondary objectives were the evaluation of: mean change in BCVA from baseline to month 6 , mean change in central macular thickness (CMT) from baseline to months 6 and 12 , time to the first retreatment and the total number of treatments, change in lesion size and morphology from screening to months 6 and 12, and safety of intravitreal injections of ranibizumab. Total VA score for each eye was recorded. OCT was performed on both eyes at each study visit prior to study-drug administration, and CMT, along with the presence or absence of intraretinal cysts and subretinal fluid was recorded. These measurements were carried out by trained personnel using one of the three eligible SD OCTs: Heidelberg (Spectralis), Topcon, or Zeiss (Cirrus), with follow-up of an individual performed on the same type of OCT. In cases of diagnostic uncertainty, OCT grading was undertaken by physicians not involved in the patient's treatment. Fluorescein angiography was performed in conjunction with colour fundus photography using standard procedures at screening, baseline, and months 6 and 12, (with further assessments at the investigator's discretion if needed). The area of the lesion, evidence and location of $\mathrm{CNV}$ relative to the fovea, the presence of fluorescein leakage, and the presence or absence of subretinal or intraretinal haemorrhage or other disease were recorded.

Exploratory end points included patient-reported outcomes from baseline to months 6 and 12 as assessed by the Macular Treatment Satisfaction Questionnaire (MacTSQ) and the 12-item Wellbeing Questionnaire (W-BQ12). The MacTSQ1020 was developed in 2006/ 2007, and is based on the Diabetes Treatment Satisfaction Questionnaire (DTSQ) and the Retinopathy Treatment Satisfaction Questionnaire (RetTSQ). ${ }^{21}$ It consists of 13 items, each scored on a 7 -point Likert scale $(6=$ very satisfied; $0=$ very dissatisfied). Adapted from a longer version (W-BQ22), the W-BQ12 is a short-form questionnaire that includes only 12 of the original 22 items. ${ }^{22}$ It has been designed to measure general wellbeing (negative wellbeing, energy, and positive wellbeing) using a balanced selection of both negative and positive items. It is widely used in clinical trials, including in patients with macular disease. ${ }^{22}$ The 12 items are scored on a scale from 0 (not at all) to 3 (all the time). The questionnaire can be scored according to the three subscales or with all 12 items to produce a score for general wellbeing (scale: 0-36, with higher values indicating greater wellbeing). With regards to safety, only serious adverse events (SAEs) were recorded for the interim analysis. 


\section{Statistical analysis}

A sample size of 58 was calculated to have $90 \%$ power to detect a difference in mean BCVA of 10 letters for the primary efficacy end point (change in BCVA from baseline to 12 months), assuming a standard deviation (SD) of differences of 23 , using a paired $t$-test with a 0.05 two-sided significance level. Sample size calculations were based on previously reported data for ranibizumab in patients with myopic CNV. ${ }^{18}$ To allow for non-completing patients, a recruitment target of 64 patients (one eye per patient) was set. An interim analysis was planned for when $75 \%$ of the patients had completed 6 months of the study in order to provide data on the short-term efficacy and safety benefit of ranibizumab treatment in myopic CNV. This analysis also provided information that could inform a strategy of guided individualized ranibizumab treatment on an ongoing basis and allow the decision of whether or not to continue the study. In addition, unlike neovascular AMD, previous case series evaluating the role of anti-VEGF in myopic $\mathrm{CNV}$ have suggested a more self-limiting disease course, and therefore a well-conducted 6-month interim end point is of clinical use to the community.

Efficacy was assessed using the Full Analysis Set (FAS) - all patients who received at least one application of study treatment and had at least one post-baseline assessment for BCVA (assessed using the lastobservation-carried-forward approach and intent-to-treat principles). Safety was assessed for all patients who received at least one application of study treatment and had at least one post-baseline safety assessment.

Descriptive statistics for absolute values and changes from baseline were reported for each end point. For the purposes of the McNemar tests, category 'Questionable' was included with the 'Definite' category. Missing values (N/A) were excluded.

\section{Results}

\section{Demographic and baseline characteristics}

In total, 65 patients were recruited at 12 investigational centres in the United Kingdom. Forty-eight patients were evaluated at the protocol-planned interim analysis when $75 \%$ of patients had completed eight visits (month 6). At this point, one patient withdrew from the study after the provision of baseline data only. Patients' demographic data, and baseline ophthalmic and lesion criteria, are presented in Table 1. Most patients were Caucasian (91.7\%), female $(72.9 \%)$, and aged $<65$ years $(75 \%)$. CNV was predominantly subfoveal $(66.7 \%)$ and was reported to have been present for a mean of 1.8 months. In $72.9 \%$ of patients, the study eye was the worst-seeing eye.

\section{Visual outcome measures}

Mean VA improved by $12.2(\mathrm{SD}=14.6)$ letters at 6 months from a baseline value of 58.8 letters $(S D=14.7)$, $P<0.001$ (Figure 2). The greatest improvement was observed in the first month of treatment (mean change, 10.2 letters, $P<0.001$ ), this increased slightly and was maintained throughout the 6-month period.

Post hoc analysis of change in BCVA of the 47 patients at 6 months demonstrated that all patients avoided a loss of $\geq 15$ letters, $98 \%$ avoided the loss of $\geq 8$ letters, and $36 \%$ gained $\geq 15$ letters.

CMT reduced by $108 \mu \mathrm{m}(\mathrm{SD}=109 \mu \mathrm{m})$ at 6 months from a mean of $366 \mu \mathrm{m}(\mathrm{SD}=113 \mu \mathrm{m})$ at baseline, $P<0.001$ in patients receiving intravitreal ranibizumab (Figure 3). A substantial improvement of $86 \mu \mathrm{m}$ $(\mathrm{SD}=95 \mu \mathrm{m}, P<0.001)$ from baseline was observed at 1 month following treatment.

Following treatment with intravitreal ranibizumab, fewer patients had intraretinal oedema with centre involvement (present in $13.0 \%$ vs absent in $82.6 \%$, data not available in $4.3 \%$ ), intraretinal cysts (present in $10.9 \%$ vs absent in $82.6 \%$, questionable in $4.3 \%$, data not available in $2.2 \%$ ), or subretinal fluid (present in $13.0 \%$ vs absent in $84.8 \%$, data not available in $2.2 \%$ ) at 6 months than at baseline, $P<0.001$ when compared to baseline for all criteria. Mean lesion size was reduced by $0.54 \mathrm{~mm}^{2}$ $(\mathrm{SD}=1.09 \mathrm{~mm})$ from $1.67-1.12 \mathrm{~mm}^{2}$ at 6 months.

Patients received a mean number of 2.9 treatments in total, and thus a mean of 1.9 retreatments was required after the prescribed first treatment (Figure 4).

\section{Patient-reported outcome measures}

In general, patients were satisfied with ranibizumab treatment, as assessed by the MacTSQ. At 6 months, the MacTSQ mean score had increased to 58.2 (maximum score: 72) from a 1-month score of 55.3, $P=0.003$. In addition, wellbeing was maintained during the 6-month period, with the W-BQ12 score rising to 25.7 at 6 months (maximum score $=35$ ) from a baseline score of 25.1, $P=0.53$.

\section{Safety}

Only SAEs were reported for the interim analysis. There was only one reported ocular SAE, a case of culture negative endophthalmitis, which was considered by investigators to be related to the injection. In addition, there were three nonocular SAEs: two hospitalizations (one because of preexisting anaemia, and the other because of pre-existing depression and anxiety) and one termination of a partner's pregnancy for social reasons. All of these non-ocular SAEs were considered by investigators as unrelated to the study medication. Overall, there were no new safety signals identified in the interim analysis. 
Table 1 Patient demographic, and baseline ophthalmic and lesion criteria, for 48 patients included in the efficacy evaluations

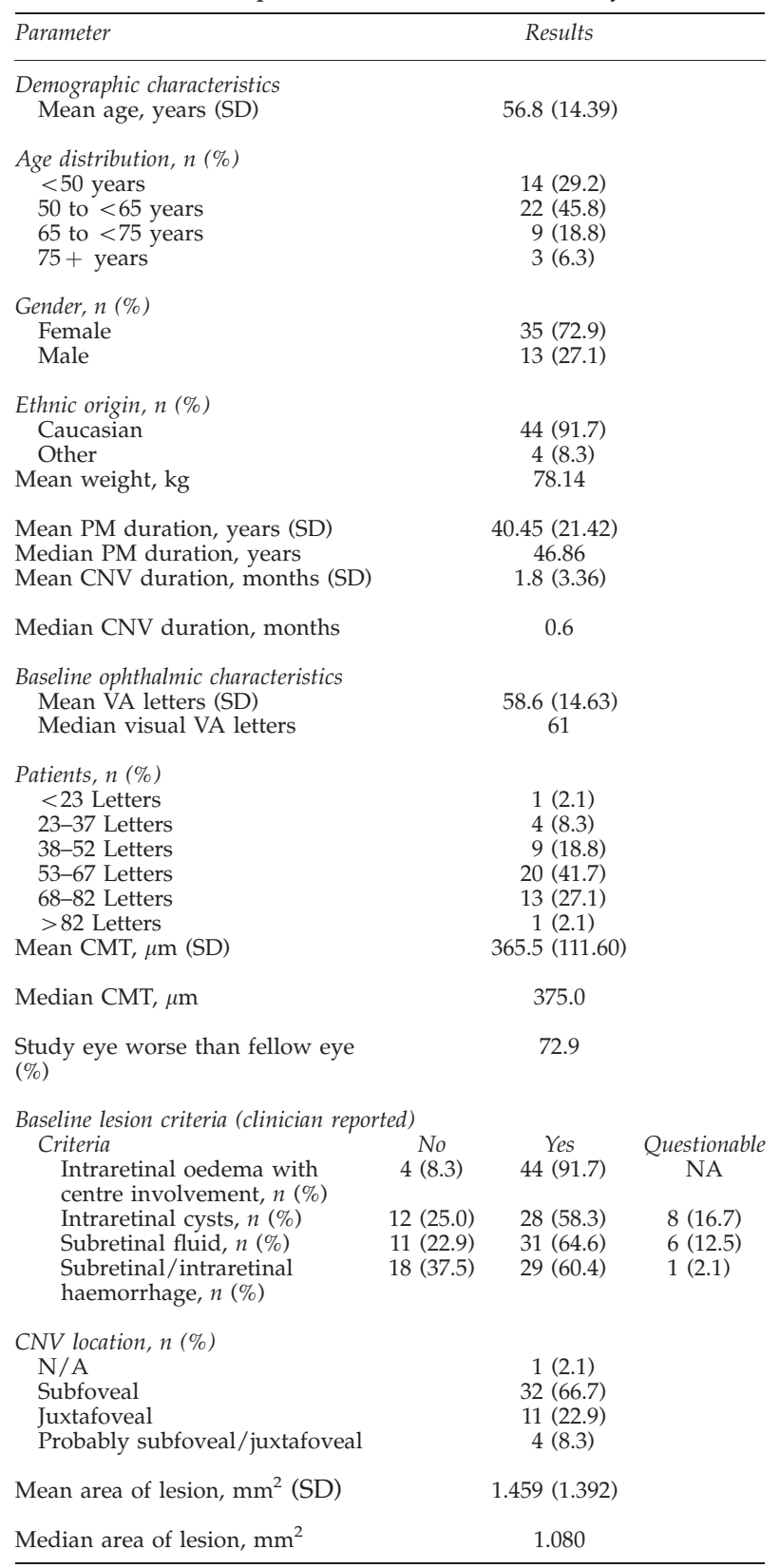

Abbreviations: CMT, central macular thickness; $\mathrm{CNV}$, choroidal neovascularisation; N/A, not available; PM, pathological myopia; SD, standard deviation; VA, visual acuity.

Baseline lesion criteria data are derived from the full safety data set.

\section{Discussion}

The 6-month planned interim analysis from the REPAIR study supports the role of ranibizumab as a beneficial treatment for myopic CNV. Ranibizumab improved VA, reduced CMT, and achieved positive changes in lesion size and morphology at 6 months compared with baseline, with few SAEs. These improvements in vision

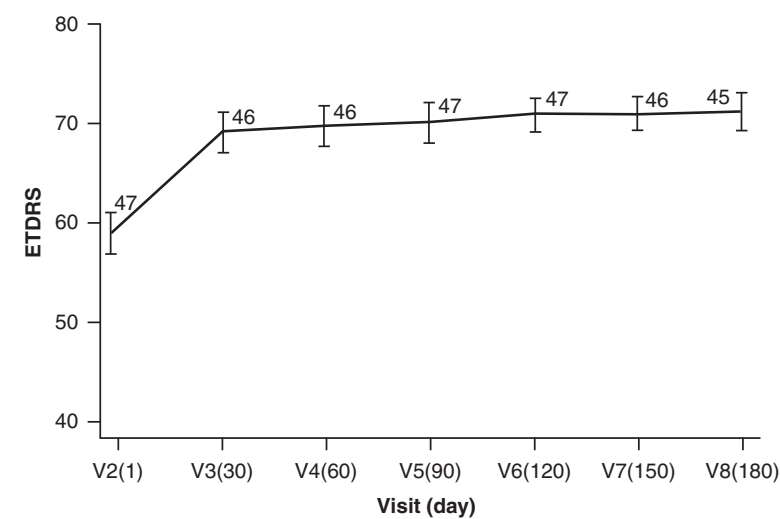

Figure 2 Mean visual acuity (letters) of the study by visit. FAS - number of patients available per data point is noted on slide. Error bars indicate \pm one SE of mean.

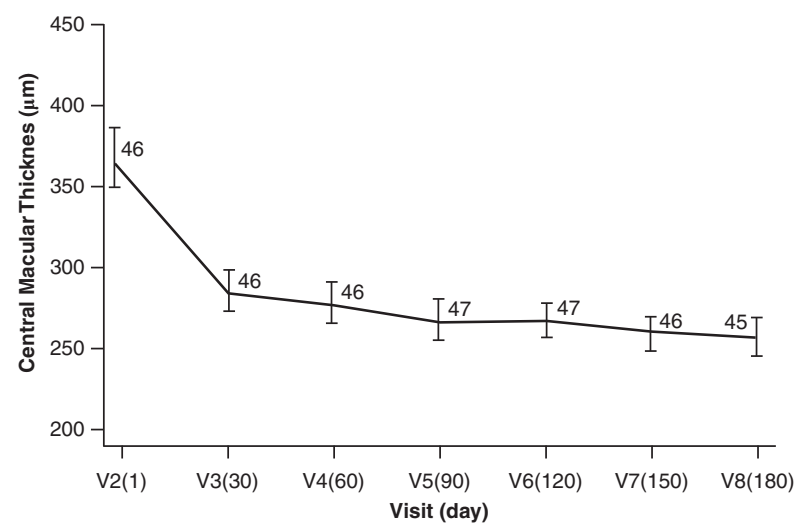

Figure 3 Mean CMT of study eye $(\mu \mathrm{m})$ by visit. FAS—number of patients available per data point is noted on slide. Error bars indicate \pm one SE of mean.

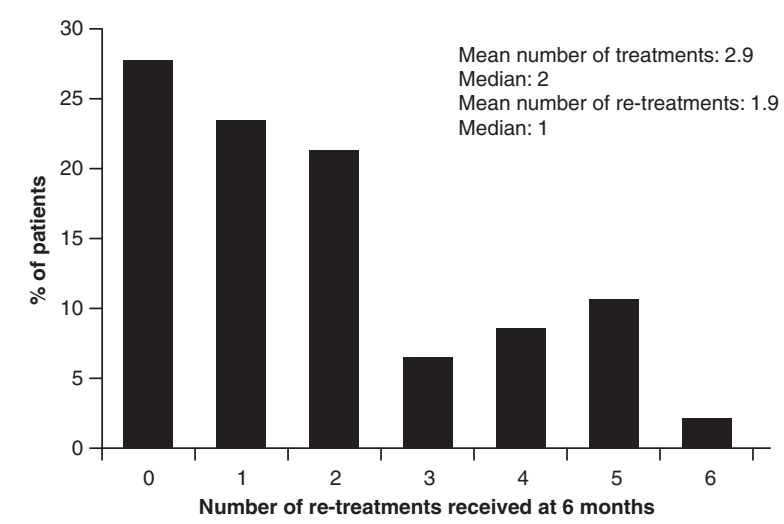

Figure 4 Number of retreatments following initial dose.

were achieved with a low number of retreatments after the initial dose. Furthermore, patients reported satisfaction with their treatment and their wellbeing was maintained. 
These data, which show an improvement in VA of 12.2 letters at 6 months compared with baseline with a mean of 2.9 injections, confirm previously published results from smaller case series reports of ranibizumab therapy in myopic CNV. These studies, although of differing designs and durations, have consistently demonstrated significant improvements in VA with ranibizumab. These studies report gains of 9-19 letters with ranibizumab therapy, $5,13,14,16-19$ and are consistent with the notion of ranibizumab's superiority to vPDT. In the VIP trial, vPDT showed stabilization of disease with a median VA loss of -1 letter at 6 months' time point compared with placebo (loss of 9 letters at 6-months) ${ }^{9,10}$ Post hoc analysis in this study found that $98 \%$ of patients avoided the loss of eight or more letters at the 6 months' time point, which compares favourably to the VIP study primary end point data previously discussed. Thus, given the substantial risk of deterioration of vision over time in untreated patients with myopic $\mathrm{CNV}^{6}$ intravitreal ranibizumab appears to offer a substantial benefit and the potential for VA recovery. Nonetheless, these ranibizumab results remain preliminary and 12 months' data are required to confirm any ongoing benefit.

Unlike CNV in neovascular AMD, myopic CNV occurs predominantly in those aged $\leq 50$ years. ${ }^{3}$ Largely affecting working aged adults, this condition has the potential to significantly limit tasks performed at work, reduce career expectations, and force early retirement, thereby having a substantial and adverse economic impact on individuals and society. ${ }^{2}$ Thus, any treatment that improves vision or prevents vision loss is likely to be economically beneficial. Although long-term efficacy data and economic analyses are required to determine the cost effectiveness of ranibizumab compared with currently approved therapies for myopic CNV, the low number of retreatments required in this study, along with the corresponding patient satisfaction with treatment, is promising.

Only SAEs were included in the interim safety analysis, which did not reveal any safety concerns with intravitreal ranibizumab in patients suffering from myopic CNV. Indeed, only one SAE (a case of culturenegative endophthalmitis) potentially related to the injection was observed. ${ }^{15}$ Furthermore, data from the current REPAIR study are consistent with the wellcharacterized safety profile demonstrated by ranibizumab in this patient population in published, smaller, less robustly designed case series. 5,13,14,16-19 Despite the potentially increased risk of retinal detachment in myopic eyes following intravitreal injections, there were no reports of serious ocular or systemic adverse events reported in these studies. Additional long-term data are needed to more fully evaluate the safety of ranibizumab in patients with myopic CNV.

The uncontrolled nature of the study design poses a limitation for the interpretation of the efficacy data in addition to the safety data. However, given that ranibizumab had already shown superior efficacy to vPDT for the treatment of $\mathrm{CNV}$ in patients with neovascular AMD, and a potential benefit for ranibizumab compared with vPDT was suggested by results from several case series in patients with myopic $\mathrm{CNV} ;{ }^{16-19}$ it was difficult to justify the allocation of patients to vPDT as a comparator arm. Furthermore, it would be challenging to recruit patients to such a study in countries where anti-VEGF therapies are available. Finally, an open-label design is likely to yield clinically meaningful results given the objective and robust nature of the primary end point of mean BCVA change from baseline.

A further limitation of this study is the lack of a central reading centre, which means that the interpretation of myopic CNV remains investigator-dependent in this study. However, given that the positive outcomes of this study are reliant on clinician-driven retreatment decisions, these results are more likely to reflect 'real world' outcomes.

In conclusion, ranibizumab may be useful in the treatment of myopic CNV. This pre-planned interim analysis of the REPAIR study, the largest study to date evaluating ranibizumab for myopic $\mathrm{CNV}$, showed that treatment with ranibizumab provides improvement in VA in this patient group. Moreover, this improvement was achieved with a low number of injections and no new safety concerns were identified. Patients were satisfied with treatment and wellbeing was maintained.

\section{Summary}

What was known before

- For a relatively young patient group, myopic CNV remains a major cause of blindness in the developed world.

- Unmet medical need exists, as current therapies such as laser and vPDT are associated with limited and disappointing results.

- Small case series utilizing anti-VEGF therapies have demonstrated encouraging results.

What this study adds

- Over a 6-month period, ranibizumab improved VA, reduced CMT, and achieved positive changes in lesion size and morphology compared with baseline criteria.

- This was achieved with a low number of retreatments after the initial dose.

- No new safety issues were identified. 


\section{Conflict of interest}

The institutes of the following authors have received funding from Novartis to cover the study-related costs: S Sivaprasad, W Amoaku, S George, A Lotery, A Browning, M McKibbin, and M Majid. W Amoaku has received support for travel to meetings in relation to this study. C Andrews, C Brittain, and A Osborne are employees of Novartis. A Tufail, PJ Patel, M Cole, R Gale, $G$ Menon, and Y Yang have no conflict of interest to disclose.

\section{Acknowledgements}

We thank Sue Harris and Sue Cheer representatives of ApotheCom for writing assistance (provision of first manuscript draft), which was funded by Novartis Pharmaceuticals Ltd. (UK). Novartis Pharmaceuticals UK Ltd., Surrey, UK participated in the design of the study, conducting the study, data collection, data management, data analysis, interpretation of the data, preparation and review of the manuscript. A Tufail, and PJ Patel have received a proportion of their funding from the Department of Health's NIHR Biomedical Research Centre for Ophthalmology at Moorfields Eye Hospital and UCL Institute of Ophthalmology. The views expressed in the publication are those of the authors (AT and PP), and not necessarily those of the Department of Health.

\section{References}

1 Montero JA, Ruiz-Moreno JM. Treatment of choroidal neovascularization in high myopia. Curr Drug Targets 2010; 11(5): 630-644.

2 Soubrane G. Choroidal neovascularization in pathologic myopia: recent developments in diagnosis and treatment. Surv Ophthalmol 2008; 53(2): 121-138.

3 Chan WM, Ohji M, Lai TYY, Liu DTL, Tano Y, Lam DSC. Choroidal neovascularisation in pathological myopia: an update in management. Br J Ophthalmol 2005; 89(11): 1522-1528

4 Battaglia Parodi M, Iacono P, Bandello F. Antivascular endothelial growth factor for choroidal neovascularization in pathologic myopia. Dev Ophthalmol 2010; 46: 73-83.

5 Cohen SY. Anti-VEGF drugs as the 2009 first-line therapy for choroidal neovascularization in pathologic myopia. Retina 2009; 29(8): 1062-1066.

6 Yoshida T, Ohno-Matsui K, Yasuzumi K, Kojima A, Shimada N, Futagami S et al. Myopic choroidal neovascularization: a 10-year follow-up. Ophthalmology 2003; 110(7): 1297-1305.

7 Ohno-Matsui K, Yoshida T, Futagami S, Yasuzumi K, Shimada N, Kojima A et al. Patchy atrophy and lacquer cracks predispose to the development of choroidal neovascularisation in pathological myopia. $\mathrm{Br} J$ Ophthalmol 2003; 87: 570-573.

8 Secretan M, Kuhn D, Soubrane G, Coscas G. Long-term visual outcome of choroidal neovascularization in pathologic myopia: natural history and laser treatment. Eur J Ophthalmol 1997; 7(4): 307-316.

9 Verteporfin in Photodynamic Therapy (VIP) Study Group. Photodynamic therapy of subfoveal choroidal neovascularization in pathologic myopia with verteporfin. 1-year results of a randomized clinical trial-VIP report no. 1. Ophthalmology 2001; 108(5): 841-852.

10 Blinder KJ, Blumenkranz MS, Bressler NM, Bressler SB, Donato G, Lewis H et al. Verteporfin in Photodynamic Therapy (VIP) Study Group. Verteporfin therapy of subfoveal choroidal neovascularization in pathologic myopia: 2-year results of a randomized clinical trial-VIP report no. 3.. Ophthalmology 2003; 110(4): 667-673.

11 Brown DM, Michels M, Kaiser P, Heier JS, Sy JP, Ianchulev T. Ranibizumab versus verteporfin photodynamic therapy for neovascular age-related macular degeneration: two-year results of the ANCHOR study. Ophthalmology 2009; 116: 57-65.

12 Tufail A, Patel P, Egan C, Hykin P, da Cruz L, Gregor Z et al. Bevacizumab for neovascular age related macular degeneration (ABC Trial): multicentre randomised double masked study. BMJ 2010; 340: c2459.

13 Ng DS, Kwok AK, Chan CW. Anti-vascular endothelial growth factor for myopic choroidal neovascularization. Clin Experiment Ophthalmol 2012; 40(1): 98-110.

14 Calvo-Gonzalez C, Reche-Frutos J, Donate J, FernandezPerez C, Garcia-Feijoo J. Intravitreal ranibizumab for myopic choroidal neovascularization: factors predictive of visual outcome and need for retreatment. Am J Ophthalmol 2011; 151: 529-534.

15 Lucentis Summary of Product Characteristics Electronic Medicines Compendium (Updated 2012; cited 26 June 2012). Available from http://www.medicines.org.uk/ $\mathrm{EMC} /$ medicine/19409/SPC/Lucentis $+10+\mathrm{mg}+\mathrm{ml}$ + solution + for + injection/.

16 Silva RM, Ruiz-Moreno JM, Nascimento J, Carneiro A, Rosa P, Barbosa A et al. Short-term efficacy and safety of intravitreal ranibizumab for myopic choroidal neovascularization. Retina 2008; 28(8): 1117-1123.

17 Konstantinidis L, Mantel I, Pournaras JA, Zografos L, Ambresin A. Intravitreal ranibizumab (Lucentis) for the treatment of myopic choroidal neovascularization. Graefes Arch Clin Exp Ophthalmol 2009; 247(3): 311-318.

18 Mones JM, Amselem L, Serrano A, Garcia M, Hijano M. Intravitreal ranibizumab for choroidal neovascularization secondary to pathologic myopia: 12-month results. Eye (Lond) 2009; 23(6): 1275-1280; quiz 81.

19 Lai TY, Chan WM, Liu DT, Lam DS. Intravitreal ranibizumab for the primary treatment of choroidal neovascularization secondary to pathologic myopia. Retina 2009; 29(6): 750-756.

20 Mitchell J, Brose LS, Bradley C. Design of a measure of satisfaction with treatment for Macular Degeneration (MacTSQ). Qual Life Res 2007, A-120(Suppl) International Society for Quality of Life Research meeting abstracts, Abstract 1150.

21 Bradley C, Lewis KS. Measures of psychological well-being and treatment satisfaction developed from the responses of people with tablet-treated diabetes. Diabet Med 1990; 7(5): 445-451.

22 Mitchell J, Bradley C. Psychometric evaluation of the 12-item Well-being Questionnaire for use with people with macular disease. Qual Life Res 2001; 10(5): 465-473. 